\title{
More transition amplitudes on the Riemann sphere
}

\author{
J. Dimock \\ Dept. of Mathematics \\ SUNY at Buffalo \\ Buffalo, NY 14260
}

November 1, 2018

\begin{abstract}
We consider a conformal field theory for bosons on the Riemann sphere. Correlation functions are defined as singular limits of functional integrals. The main result is that these amplitudes define transition amplitudes, that is multilinear Hilbert-Schmidt functionals on a fixed Hilbert space.
\end{abstract}

\section{Introduction}

We consider a bosonic conformal field theory on the Riemann sphere $\mathbb{C}_{\infty}=\mathbb{C} \cup\{\infty\}$. The theory is determined by correlation functions which are formally defined as follows. Let $\gamma$ a metric on $\mathbb{C}_{\infty}$ of the form $\gamma=\rho|d z|^{2}$. Let the fields $X(z, \bar{z})$ be Gaussian random variables with covariance which is the kernel of $2 \pi\left(-\Delta_{\gamma}\right)^{-1}$ where $\Delta_{\gamma}$ is the Laplacian for this metric. Consider the derivatives $\partial^{m} X(z)$ of order $m=1,2,3, \ldots$. Then the correlations are the expectations

$$
<\partial^{m_{1}} X\left(z_{1}\right) \cdots \partial^{m_{n}} X\left(z_{n}\right)>
$$

This can be made precise if the fields are regarded as distributions. If one avoids coinciding points the correlation functions are also well-defined at sharp points.

Our goal in this paper is to give a precise definition of the Hilbert space structure associated with such correlation functions. This comes in two stages

In the first stage (sections 2- 5) we consider fields localized in the disc $D=\{z:|z|<1\}$. We show that associated correlation functions have a positivity property under reflections through the circle $|z|=1$. The reflection positivity leads to the construction of a Hilbert space $\mathcal{H}$ by standard methods. We develop the structure of this Hilbert space at some length, and in particular identify it as a Fock space.

In the second stage (section 6) we consider a family of disjoint discs $\left\{D_{k}\right\}$ in $\mathbb{C}_{\infty}$ and correlation functions with fields in the discs. We show via parametrizations $D \rightarrow D_{k}$ that the correlation functions define a multilinear functionals on $\mathcal{H}$, i.e. they determine transition amplitudes. In fact we obtain the stronger result that they are Hilbert-Schmidt functionals. This is the main result.

In an earlier paper [2] we considered this problem for fields $\left[e^{i k X(z, \bar{z})}\right]_{r}$, a regularization of $e^{i k X(z, \bar{z})}$. In this case similar but substantially weaker results were obtained.

Our results are likely to be useful for establishing sewing properties. For this one would want to parametrize some of the discs by $D^{\prime}=\{z:|z|>1\}$ rather than $D$. For progress in this direction see [1], 2], [5].

This work is also pointing toward establishing similar results in string theory, i.e defining string theory scattering amplitudes as multilinear functionals on a full Fock space. At the end of the paper we comment on the work to be done to accomplish this goal. 


\section{The basic construction}

\section{1}

In this section we define the model and verify that it has the standard features of a conformal field theory. Axiomatic treatments of conformal field theory can be found in [3, 4], 7].

We generally work in the subset $\mathbb{C} \subset \mathbb{C}_{\infty}$ with standard coordinates. These are denoted $x=\left(x^{1}, x^{2}\right)$ or $z=x^{1}+i x^{2}, \bar{z}=x^{1}-i x^{2}$. We consider conformal metrics

$$
\gamma=\rho|d z|^{2}=\rho\left(\left(d x^{1}\right)^{2}+\left(d x^{2}\right)^{2}\right)
$$

For such metrics

$$
|\gamma(x)| \equiv \operatorname{det}\left\{\gamma_{a b}(x)\right\}=\rho(x)^{2}
$$

and we have the associated volume form or measure

$$
|\gamma(z)|^{1 / 2} \frac{i}{2} d z \wedge d \bar{z}=|\gamma(x)|^{1 / 2} d x=\rho(x) d x
$$

Derivatives are denoted $\partial_{a}=\partial / \partial x^{a}$ and $\partial=\frac{1}{2}\left(\partial_{1}-i \partial_{2}\right)$ and $\bar{\partial}=\frac{1}{2}\left(\partial_{1}+i \partial_{2}\right)$. The Laplacian is

$$
\Delta_{\gamma}=\frac{4}{\rho} \bar{\partial} \partial=\frac{1}{\rho}\left(\partial_{1}^{2}+\partial_{2}^{2}\right)
$$

The Laplacian is self-adjoint on the complex Hilbert space $L^{2}(\mathbb{C}, \rho d x)$. The Laplacian vanishes on the constants and we consider the subspace $\{1\}^{\perp}$ of functions orthogonal to constants, that is functions for which $\int f(x) \rho(x) d x=0$. The Laplacian is invertible on this subspace. The inverse can be related to the inverse for the flat metric and for $f, h \in\{1\}^{\perp}$ one finds

$$
2 \pi\left(f,\left(-\Delta_{\gamma}\right)^{-1} h\right)=\int \overline{f(x)} \rho(x) \log \left|x-x^{\prime}\right| \rho\left(x^{\prime}\right) h\left(x^{\prime}\right) d x d x^{\prime}
$$

Now let $X(f)$ be a family of Gaussian random variables indexed by real functions $f \in\{1\}^{\perp}$ with mean zero and covariance $2 \pi\left(-\Delta_{\gamma}\right)^{-1}$. Expectations are denoted by $<\cdot>_{\gamma}$ so we have the two point function

$$
<X(f) X(h)>_{\gamma}=2 \pi\left(f,\left(-\Delta_{\gamma}\right)^{-1} h\right)
$$

The correlation functions for $X(f)$ satisfy a basic reflection positivity property. Let $\theta$ be reflection through the circle $|z|=1$, i.e. $\theta(z)=\bar{z}^{-1}$. Suppose that the metric $\gamma=\rho|d z|^{2}$ is chosen so reflection is an isometry. i.e $\rho$ must satisfy $\rho(z)=|z|^{-4} \rho\left(\bar{z}^{-1}\right)$. Then $\theta$ induces an anti-unitary map $\Theta$ on the underlying complex $L^{2}$ space such that

$$
\Theta\left(X\left(f_{1}\right) \ldots X\left(f_{n}\right)\right)=X\left(\theta^{*} f_{1}\right) \ldots X\left(\theta^{*} f_{n}\right)
$$

where $\theta^{*} f=f \circ \theta$. Then if $F$ is any polynomial in $X(f)$ or more generally any $L^{2}$ function we have the positivity

$$
<\Theta(F) F>_{\gamma} \geq 0
$$

This is proved in 2] by using an approximation by massive fields and a Markov property. An alternative would be to give a basic Fock space construction of the correlation functions in which case the positivity would be obvious. (We obtain Fock space as a derived object later on.) 


\section{2}

Now we want to consider fields at sharp points. The field $X(z, \bar{z})$ is not well-defined but the derivatives $\partial X(z)$ are as we now explain

A delta function at $z \in \mathbb{C}$ is the distribution which sends the test function $f$ to $\left\langle\delta_{z}, f\right\rangle=f(z)$. We want to approximate it by functions on $\mathbb{C}$. With the metric $\gamma=\rho|d z|^{2}$ a function $h$ defines a distribution by $h \mapsto<h, f>_{\rho}$ where

$$
<h, f>_{\rho}=\int h(x) f(x) \rho(x) d x
$$

Let $\delta_{\kappa}(\cdot-z)$ be a family of smooth functions with shrinking supports converging to $\delta_{z}$ function in the flat metric $|d z|^{2}$. Then $\rho^{-1} \delta_{\kappa}(\cdot-z)$ converges to the delta function in the metric $\rho|d z|^{2}$ for we have

$$
\lim _{\kappa \rightarrow \infty}<\rho^{-1} \delta_{\kappa}(\cdot-z), f>_{\rho}=\lim _{\kappa \rightarrow \infty}<\delta_{\kappa}(\cdot-z), f>_{1}=f(z)
$$

The derivative of a delta function at $z \in \mathbb{C}_{\infty}$ is the map taking smooth functions $f$ on $\mathbb{C}_{\infty}$ to $(\partial f)(z)$. Then $\rho^{-1}(-\partial) \delta_{\kappa}(\cdot-z)$ is an approximation for we have

$$
\lim _{\kappa \rightarrow \infty}<\rho^{-1}(-\partial) \delta_{\kappa}(\cdot-z) f>_{\rho}=\lim _{\kappa \rightarrow \infty}<\delta_{\kappa}(\cdot-z), \partial f>_{1}=\partial f(z)
$$

Thus as an approximation to $\partial X(z)$ we consider fields $X\left(\rho^{-1}(-\partial) \delta_{\kappa}(\cdot-z)\right)$. Note that the function $\rho^{-1}(-\partial) \delta_{\kappa}(\cdot-z)$ is orthogonal to constants so this is well-defined.

Theorem 1 For conformal metrics $\gamma=\rho|d z|^{2}$

1. If $z \neq z^{\prime}$ the limit

$$
C\left(z, z^{\prime}\right)=\lim _{\kappa \rightarrow \infty}\left\langle X\left(\rho^{-1}(-\partial) \delta_{\kappa}(\cdot-z)\right) X\left(\rho^{-1}(-\partial) \delta_{\kappa}\left(\cdot-z^{\prime}\right)\right)\right\rangle_{\gamma}
$$

exists, is independent of $\gamma$, and is given by

$$
C\left(z, z^{\prime}\right)=-\frac{1}{2} \frac{1}{\left(z-z^{\prime}\right)^{2}}
$$

2. For non-coinciding points $z_{1}, \ldots, z_{n}$ the limit

$$
C\left(z_{1}, \ldots, z_{n}\right)=\lim _{\kappa \rightarrow \infty}\left\langle X\left(\rho^{-1}(-\partial) \delta_{\kappa}\left(\cdot-z_{1}\right)\right) \cdots X\left(\rho^{-1}(-\partial) \delta_{\kappa}\left(\cdot-z_{n}\right)\right)\right\rangle_{\gamma}
$$

exists and is independent of $\gamma$. It vanishes if $n$ is odd and if $n$ is even is given by

$$
C\left(z_{1}, \ldots, z_{n}\right)=\sum_{P} \prod_{\{\alpha, \beta\} \in P} C\left(z_{\alpha}, z_{\beta}\right)
$$

where the sum is over all pairings $P=\left\{\left\{\alpha_{1}, \beta_{1}\right\}, \ldots,\left\{\alpha_{n / 2}, \beta_{n / 2}\right\}\right\}$ of $(1, \ldots, n)$.

Remark. The function $C\left(z_{1}, \ldots, z_{n}\right)$ gives a meaning to the formal expression $<\partial X\left(z_{1}\right) \cdots \partial X\left(z_{n}\right)>$. Note that it is symmetric and analytic away from coinciding points.

Proof. We have

$$
\partial_{z} \partial_{z^{\prime}}\left(-\log \left|z-z^{\prime}\right|\right)=-\frac{1}{2} \frac{1}{\left(z-z^{\prime}\right)^{2}}=C\left(z, z^{\prime}\right)
$$


and so by (6) with $d^{2} w=d(\operatorname{Re} w) d(\operatorname{Im} w)$

$$
\begin{aligned}
& \left\langle X\left(\rho^{-1}(-\partial) \delta_{\kappa}(\cdot-z)\right) X\left(\rho^{-1}(-\partial) \delta_{\kappa}\left(\cdot-z^{\prime}\right)\right)\right\rangle \\
= & \int\left(-\partial_{w} \delta_{\kappa}(w-z)\right)\left(-\log \left|w-w^{\prime}\right|\right)\left(-\partial_{w^{\prime}} \delta_{\kappa}\left(w^{\prime}-z^{\prime}\right)\right) d^{2} w d^{2} w^{\prime} \\
= & \left.\int \delta_{\kappa}(w-z)\right) C\left(w, w^{\prime}\right) \delta_{\kappa}\left(w^{\prime}-z^{\prime}\right) d^{2} w d^{2} w^{\prime}
\end{aligned}
$$

This converges to $C\left(z, z^{\prime}\right)$. For the second part the regularized expression is a standard Gaussian integral. It is evaluated as (16) with $C\left(z, z^{\prime}\right)$ replaced by (18). The result follows by taking the limit.

The correlation functions are so far only defined in the coordinate patch $\mathbb{C}$ and we add some remarks on including the point at infinity. For this we go to the other coordinate patch on $\mathbb{C}_{\infty}-\{0\}$ with coordinates $\zeta=1 / z$. The metric is now $\hat{\gamma}=\hat{\rho}|d \zeta|^{2}$ where

$$
\hat{\rho}(\zeta)=\rho(1 / \zeta) \frac{d z}{d \zeta} \frac{d \bar{z}}{d \bar{\zeta}}=\rho(1 / \zeta)|\zeta|^{-4}
$$

With $\hat{f}(\zeta)=f(1 / \zeta)$ the expression (6) becomes in new coordinates

$$
2 \pi\left(f,\left(-\Delta_{\gamma}\right)^{-1} h\right)=\int \overline{\hat{f}(\zeta)} \hat{\rho}(\zeta)\left(-\log \left|\frac{1}{\zeta}-\frac{1}{\zeta^{\prime}}\right|\right) \hat{\rho}\left(\zeta^{\prime}\right) \hat{h}\left(\zeta^{\prime}\right) d^{2} \zeta d^{2} \zeta^{\prime}
$$

Now let $\hat{f}=\hat{\rho}^{-1}(-\partial) \delta(\cdot-\zeta)$ and $\hat{h}=\hat{\rho}^{-1}(-\partial) \delta\left(\cdot-\zeta^{\prime}\right)$ and take the limit $\kappa \rightarrow \infty$ to get the two point function in these coordinates. We find for $\zeta, \zeta^{\prime} \in \mathbb{C}$

$$
\hat{C}\left(\zeta, \zeta^{\prime}\right)=-\frac{1}{2} \frac{1}{\left(\zeta-\zeta^{\prime}\right)^{2}}
$$

Note that in each variable the correlation functions transform as one-forms for we have on $\mathbb{C}-\{0\}$

$$
\hat{C}\left(\zeta, \zeta^{\prime}\right)=-\frac{1}{2} \frac{1}{\left(\zeta-\zeta^{\prime}\right)^{2}}=C\left(\frac{1}{\zeta}, \frac{1}{\zeta^{\prime}}\right) \frac{d z}{d \zeta} \frac{d z^{\prime}}{d \zeta^{\prime}}
$$

Similarly if only one point is expressed in new coordinates we find

$$
\tilde{C}\left(z, \zeta^{\prime}\right)=\frac{1}{2} \frac{1}{\left(1-z \zeta^{\prime}\right)^{2}}=C\left(z, \frac{1}{\zeta^{\prime}}\right) \frac{d z^{\prime}}{d \zeta^{\prime}}
$$

Returning to standard coordinates let us also note the transformation properties under general Mobius transformations

$$
w=\alpha(z)=\frac{a z+b}{c z+d}
$$

and radial reflections.

\section{Lemma 1}

For a Mobius transformation $w_{i}=\alpha\left(z_{i}\right)$

$$
C\left(z_{1}, \cdots, z_{n}\right)=C\left(w_{1}, \cdots, w_{n}\right) \prod_{i} \frac{\partial w}{\partial z}\left(z_{i}\right)
$$

Under radial reflections $\theta z=1 / \bar{z}$

$$
\overline{C\left(z_{1}, \cdots, z_{n}\right)}=C\left(\frac{1}{\bar{z}_{1}}, \cdots, \frac{1}{\bar{z}_{n}}\right) \prod_{i} \frac{-1}{\bar{z}_{i}^{2}}
$$




\begin{tabular}{||l|l||}
\hline formal random variable & symbol \\
\hline \hline$\partial X\left(z_{1}\right) \ldots \partial X\left(z_{n}\right)$ & {$\left[z_{1}, \ldots, z_{n}\right] \in \Upsilon$} \\
\hline$\partial^{m_{1}} X\left(z_{1}\right) \ldots \partial^{m_{n}} X\left(z_{n}\right)$ & {$\left[m_{1}, z_{1}, \ldots, m_{n}, z_{n}\right] \in \Upsilon^{\prime}$} \\
\hline$: \partial^{m_{1}} X\left(z_{1}\right) \ldots \partial^{m_{n}} X\left(z_{n}\right):$ & $:\left[m_{1}, z_{1}, \ldots, m_{n}, z_{n}\right]:_{0} \in \Upsilon^{\prime}$ \\
\hline$: \partial^{m_{1}} X\left(z_{1}\right) \ldots \partial^{m_{n}} X\left(z_{n}\right):$ & $:\left[m_{1}, z_{1}, \ldots, m_{n}, z_{n}\right]: \in \Upsilon^{w}$ \\
\hline
\end{tabular}

Table 1: Formal random variables are represented by symbols which are well-defined elements of the various algebras $\Upsilon, \Upsilon^{\prime}, \Upsilon^{w}$

Proof. It suffices to show these results for the two point function. In the first case for $w=\alpha(z), w^{\prime}=$ $\alpha\left(z^{\prime}\right)$

$$
C\left(z, z^{\prime}\right)=\frac{\partial w}{\partial z} \frac{\partial w^{\prime}}{\partial z^{\prime}} C\left(w, w^{\prime}\right)
$$

If it holds for Mobius transformations $\alpha, \beta$ then it holds for the composition $\alpha \circ \beta$. But any Mobius is a composition of translations, scalings, and inversions. Thus it suffices to check for each of these separately. This is easy for $w=z+b$ and $w=q z$ and for $w=1 / z$ we have have already checked it.

For reflections we have

$$
C\left(\frac{1}{\bar{z}}, \frac{1}{\bar{z}^{\prime}}\right) \frac{-1}{\bar{z}^{2}} \frac{-1}{\bar{z}^{\prime 2}}=-\frac{1}{2} \frac{1}{\left(\bar{z}-\bar{z}^{\prime}\right)^{2}}=\overline{C\left(z, z^{\prime}\right)}
$$

This completes the proof.

\section{3}

We also need a reflection positivity property. To formulate it and for future purposes we reformulate our results in a more algebraic language as in [2].

Let $\Upsilon$ be the free algebra generated by the sequences $Z=\left[z_{1}, \ldots, z_{n}\right]$ with $z_{i} \in \mathbb{C}$. We include the empty sequence. The general element is a finite sum

$$
F=\sum_{Z} F(Z) Z \quad F(Z) \in \mathbb{C}
$$

and multiplication $Z Z^{\prime}$ is juxtaposition. Let $\Upsilon_{0}$ be the subspace spanned by elements $Z$ with noncoinciding points. We define a linear function on $F \in \Upsilon_{0}$ by

$$
<F>=\sum_{Z} F(Z)<Z>
$$

where

$$
<Z>=<\left[z_{1}, \ldots, z_{n}\right]>=C\left(z_{1}, \ldots, z_{n}\right)
$$

Thus $\left[z_{1}, \ldots, z_{n}\right]$ is a symbol for the formal random variable $\partial X\left(z_{1}\right) \cdots \partial X\left(z_{n}\right)$. In table 1 we list the conventions of this type that we employ. 
Now define radial reflection on symbols $Z=\left[z_{1}, \ldots, z_{n}\right]$ with $z_{i} \neq 0$ by

$$
\Theta Z=\Theta\left[z_{1}, \ldots, z_{n}\right]=\prod_{i=1}^{n} \frac{-1}{\bar{z}_{i}^{2}}\left[\frac{1}{\bar{z}_{1}}, \cdots, \frac{1}{\bar{z}_{n}}\right]
$$

Then for non-coinciding points we have by (26) that $\overline{\langle Z\rangle}=\langle\Theta Z\rangle$. We extend $\Theta$ to the subspace of $\Upsilon$ spanned by symbols with $z_{i} \neq 0$ by defining for $F=\sum_{Z} F(Z) Z$

$$
\Theta F=\sum_{Z} \overline{F(Z)} \Theta Z
$$

Then for non-coinciding points $\overline{\langle F\rangle}=<\Theta F>$. The operator $\Theta$ is an anti-linear automorphism satisfying $\Theta^{2}=1$.

Next let $D, D^{\prime}$ be half-spheres (discs)

$$
D=\{z \in \mathbb{C}:|z|<1\} \quad D^{\prime}=\{z \in \mathbb{C}:|z|>1\}
$$

We are particularly interested in symbols $Z=\left[z_{1}, \ldots, z_{n}\right]$ with non-coinciding points and $z_{i} \in D-\{0\}$. These span a subspace of $\Upsilon_{0}$ denoted $\Upsilon_{0, D-\{0\}}$. Similarly $\Upsilon_{0, D^{\prime}}$ has non-coinciding points in $D^{\prime}$. Note that if $F, G \in \Upsilon_{0, D-\{0\}}$ then $\Theta F \in \Upsilon_{0, D^{\prime}}$ and $(\Theta F) G \in \Upsilon_{0}$.

Lemma 2 For $F, G \in \Upsilon_{0, D-\{0\}}$

$$
<(\Theta F) G>=\overline{<(\Theta G) F>}
$$

and we have the reflection positivity

$$
<(\Theta F) F>\geq 0
$$

Proof. The first identity follows from $\overline{\langle F\rangle}=<\Theta F>$ and $\Theta^{2}=1$ and the fact that $\Theta$ is an automorphism.

For the positivity we have to show that

$$
\sum_{Z, W} \overline{F(Z)} F(W)<(\Theta Z) W>\geq 0
$$

Choosing a reflection invariant metric $\gamma=\rho|d z|^{2}$ we have for $Z=\left[z_{1}, \ldots, z_{n}\right]$ and $W=\left[w_{1}, \ldots, w_{m}\right]$

$$
\begin{aligned}
<(\Theta Z) W> & =\prod_{i} \frac{-1}{\bar{z}_{i}^{2}}<\left[\bar{z}_{1}^{-1}, \ldots, \bar{z}_{n}^{-1}, w_{1}, \ldots, w_{m}\right]> \\
& =\lim _{\kappa \rightarrow \infty}\left\langle\prod_{i=1}^{n} X\left(\frac{-1}{\bar{z}_{i}^{2}} \rho^{-1}(-\partial) \delta_{\kappa}\left(\cdot-\bar{z}_{i}^{-1}\right)\right) \prod_{j=1}^{m} X\left(\rho^{-1}(-\partial) \delta_{\kappa}\left(\cdot-w_{j}\right)\right)\right\rangle_{\gamma}
\end{aligned}
$$

The reason this works is that $<\rho^{-1}(-\partial) \delta_{\kappa}\left(\cdot-\bar{z}^{-1}\right), f>_{\rho}$ converges to $\partial f\left(\bar{z}^{-1}\right)$. But we can replace $\rho^{-1}(-\partial) \delta_{\kappa}\left(\cdot-\bar{z}^{-1}\right)$ with another approximating sequence and we take $-\bar{z}^{2} \theta^{*}\left(\rho^{-1}(-\bar{\partial}) \delta_{\kappa}(\cdot-z)\right)$. This works since $\theta$ is an isometry and so

$$
\begin{aligned}
<\theta^{*}\left(\rho^{-1}(-\bar{\partial}) \delta_{\kappa}(\cdot-z), f>_{\rho}\right. & =<\rho^{-1}(-\bar{\partial}) \delta_{\kappa}(\cdot-z), \theta^{*} f>_{\rho} \\
& =\int \delta_{\kappa}(w-z) \partial_{\bar{w}} f\left(\bar{w}^{-1}\right) d z \\
& \left.=\int \delta_{\kappa}(w-z)\left(-\bar{w}^{-2}\right) \partial f\left(\bar{w}^{-1}\right)\right) d z
\end{aligned}
$$


which converges to $-\bar{z}^{-2} \partial f\left(\bar{z}^{-1}\right)$. Thus we have

$$
\begin{aligned}
\langle(\Theta Z) W\rangle & =\lim _{\kappa \rightarrow \infty}\left\langle\prod_{i=1}^{n} X\left(\theta^{*}\left(\rho^{-1}(-\bar{\partial}) \delta_{\kappa}\left(\cdot-z_{i}\right)\right)\right) \prod_{j=1}^{m} X\left(\rho^{-1}(-\partial) \delta_{\kappa}\left(\cdot-w_{j}\right)\right)\right\rangle_{\gamma} \\
& =\lim _{\kappa \rightarrow \infty}\left\langle\Theta\left(\prod_{i=1}^{n} X\left(\left(\rho^{-1}(-\partial) \delta_{\kappa}\left(\cdot-z_{i}\right)\right)\right)\right) \prod_{j=1}^{m} X\left(\rho^{-1}(-\partial) \delta_{\kappa}\left(\cdot-w_{j}\right)\right)\right\rangle_{\gamma}
\end{aligned}
$$

where now $\Theta$ is the operator on random variables defined in (8). The positivity for $\kappa<\infty$ now follows by the positivity result for random variables quoted in (9), and hence it also holds in the limit.

\section{4}

The reflection positivity gives a Hilbert space structure as follows. Let $\mathcal{N}$ be the null space all $F$ in $\Upsilon_{0, D-\{0\}}$ such that $<(\Theta F) F>=0$. By theorem $2<(\Theta F) G>$ determines an inner product on $\Upsilon_{0, D-\{0\}} / \mathcal{N}$. If $\nu_{0}$ is the mapping from $\Upsilon_{0, D-\{0\}}$ to its equivalence class in $\Upsilon_{0, D-\{0\}} / \mathcal{N}$ then $\left(\nu_{0}(F), \nu_{0}(G)\right)=<(\Theta F) G>$. The completion in the norm $\left\|\nu_{0}(F)\right\|=<(\Theta F) F>^{1 / 2}$ is then a Hilbert space

$$
\mathcal{H}=\overline{\Upsilon_{0, D-\{0\}} / \mathcal{N}}
$$

Thus we have

Theorem 2 There exists a complex Hilbert space $\mathcal{H}$ and a linear mapping $\nu_{0}: \Upsilon_{0, D-\{0\}} \rightarrow \mathcal{H}$ with dense range such that for all $F, G \in \Upsilon_{0, D-\{0\}}$

$$
\left(\nu_{0}(F), \nu_{0}(G)\right)=<(\Theta F) G>
$$

Corollary 1 The function $\nu_{0}\left(\left[z_{1}, \ldots, z_{n}\right]\right)$ is strongly analytic $z_{i} \in D-\{0\}$ away from coinciding points. It is symmetric under permutations of the $z_{i}$.

Proof. The expression

$$
\left(\nu_{0}\left(\left[w_{1}, \ldots, w_{r}\right]\right), \nu_{0}\left(\left[z_{1}, \ldots, z_{n}\right]\right)\right)=\prod_{i} \frac{-1}{\bar{w}_{i}^{2}}<\left[\bar{w}_{1}^{-1}, \ldots, \bar{w}_{r}^{-1}, z_{1}, \ldots, z_{n}\right]>
$$

is analytic in $z_{i} \in D-\{0\}$ away from coinciding points since the $\bar{w}_{j}^{-1}$ are in $D^{\prime}$ and the correlation functions have this property. Hence $\left(\nu_{0}(F), \nu_{0}\left(\left[z_{1}, \ldots, z_{n}\right]\right)\right)$ is analytic for all $F \in \Upsilon_{0, D-\{0\}}$. The $\nu_{0}(F)$ are a dense set so $\nu_{0}\left(\left[z_{1}, \ldots, z_{n}\right]\right)$ is weakly analytic and hence strongly analytic. The symmetry follows similarly.

Remark. One can show that For $z \in D-\{0\}$ there exists an operator $\partial \hat{X}(z)$ defined on the span of $\nu_{0}\left(\left[z_{1}, \ldots, z_{n}\right]\right)$ with $\left|z_{i}\right|<|z|$ such that

$$
\partial \hat{X}(z) \nu_{0}\left(\left[z_{1}, \ldots, z_{n}\right]\right)=\nu_{0}\left(\left[z, z_{1}, \ldots, z_{n}\right]\right)
$$

If we let $\Omega=\nu_{0}(\emptyset)$ then we have for $0<\left|z_{n}\right|<\cdots<\left|z_{1}\right|<1$

$$
\nu_{0}\left(\left[z_{1}, \ldots, z_{n}\right]\right)=\partial \hat{X}\left(z_{1}\right) \cdots \partial \hat{X}\left(z_{n}\right) \Omega
$$

We will not need these field operators directly, see Gawedski 4 for details. We will however treat the closely related creation and annihilation operators. 


\section{5}

We can make a similar construction based on any disc in $\mathbb{C}$, see also [3]. Consider a disc of the form

$$
\tilde{D}=\{z \in \mathbb{C}:|z-a|<R\}
$$

Radial reflection is now defined by

$$
\tilde{\theta}(a+z)=a+R^{2} / \bar{z}
$$

An anti-linear automorphism $\tilde{\Theta}$ on $\Upsilon_{0, \mathbb{C}-\{a\}}$ is defined by

$$
\tilde{\Theta}\left[a+z_{1}, \ldots, a+z_{n}\right]=\prod_{i}\left(-R^{2} / \bar{z}_{i}^{2}\right)\left[a+R^{2} / \bar{z}_{1}, \ldots, a+R^{2} / \bar{z}_{n}\right]
$$

This satisfies $\tilde{\Theta}^{2}=I$ and and for $F, G \in \Upsilon_{0, \tilde{D}-\{a\}}$

$$
<(\Theta F) G>\overline{<(\Theta G) F>} \quad<(\Theta F) F>\geq 0
$$

We then create a Hilbert space

$$
\mathcal{H}_{\tilde{D}}=\overline{\Upsilon_{0, \tilde{D}-\{a\}} / \mathcal{N}}
$$

as before. If $\tilde{\nu}$ maps to equivalence classes then $(\tilde{\nu}(F), \tilde{\nu}(G))=<(\Theta F) G>$.

We identify the spaces $\mathcal{H}, \mathcal{H}_{\tilde{D}}$ as follows. For any $a \in \mathbb{C}, q \in \mathbb{C}-\{0\}$ define an isomorphism $J$ on $\Upsilon$ by

$$
J\left[z_{1}, \ldots, z_{n}\right]=q^{n}\left[a+q z_{1}, \ldots, a+q z_{n}\right]
$$

This is normalized so that $\langle J Z\rangle=\langle Z\rangle$ and hence $\langle J F\rangle=\langle F\rangle$ for $F \in \Upsilon_{0}$. If $|q|=R$ then $J$ maps $\Upsilon_{0, D-\{0\}}$ onto $\Upsilon_{0, \tilde{D}-\{a\}}$ and satisfies $\tilde{\Theta} J=J \Theta$. Furthermore $J$ preserves inner products since for $F, G \in \Upsilon_{0, D-\{0\}}$

$$
<(\tilde{\Theta} J F) J G>=<(J \Theta F) J G>=<(\Theta F) G>
$$

Hence $J$ determines a unitary operator $\mathcal{J}: \mathcal{H} \mapsto \mathcal{H}_{\tilde{D}}$ such that

$$
\mathcal{J} \nu_{0}(F)=\tilde{\nu}(J F)
$$

There is still some arbitrariness associated with the choice of $q$ with $|q|=R$. Making a choice of $q$ is choosing a parametrization of $\tilde{D}$.

\section{More derivatives}

\section{1}

Now we add more derivatives. Since $C\left(z_{1}, \ldots, z_{n}\right)$ is analytic away from coinciding points we can define symmetric analytic functions by

$$
C\left(m_{1}, z_{1}, \ldots, m_{n}, z_{n}\right)=\partial_{z_{1}}^{m_{1}-1} \cdots \partial_{z_{n}}^{m_{n}-1} C\left(z_{1}, \ldots, z_{n}\right)
$$

This gives a meaning to the formal expression $<\partial^{m_{1}} X\left(z_{1}\right) \ldots \partial^{m_{n}} X\left(z_{n}\right)>$. By (16) this is evaluated as

$$
C\left(m_{1}, z_{1}, \ldots, m_{n}, z_{n}\right)=\sum_{P} \prod_{\{\alpha, \beta\} \in P} C\left(m_{\alpha}, z_{\alpha}, m_{\beta}, z_{\beta}\right)
$$

where

$$
C\left(m_{1}, z_{1}, m_{2}, z_{2}\right)=-\frac{1}{2} \partial_{z_{1}}^{m_{1}-1} \partial_{z_{2}}^{m_{2}-1} \frac{1}{\left(z_{1}-z_{2}\right)^{2}}=\frac{1}{2} \frac{\left(m_{1}+m_{2}-1\right) !(-1)^{m_{1}}}{\left(z_{1}-z_{2}\right)^{m_{1}+m_{2}}}
$$


These correlation functions can also be obtained as limits of more regular objects as in theorem 1

Mobius transformation have a complicated effect on these correlations. (The $\partial^{m} X(z)$ are not primary fields). Still there is a simple scaling property. For $q, a \in \mathbb{C}$

$$
C\left(m_{1}, z_{1}, \ldots, m_{n}, z_{n}\right)=C\left(m_{1}, q z_{1}+a, \ldots, m_{n}, q z_{n}+a\right) \prod_{i} q^{m_{i}}
$$

Radial reflections also have a complicated effect, but in this case we need an explicit expression. We still expect the effect on correlation functions to be complex conjugation so to find a candidate we compute

$$
\begin{aligned}
\overline{C\left(m_{1}, z_{1}, \ldots, m_{n}, z_{n}\right)} & =\partial_{\bar{z}_{1}}^{m_{1}-1} \cdots \partial_{\bar{z}_{n}}^{m_{n}-1} \overline{C\left(z_{1}, \ldots, z_{n}\right)} \\
& =\partial_{\bar{z}_{1}}^{m_{1}-1} \cdots \partial_{\bar{z}_{n}}^{m_{n}-1}\left(\prod_{j}-\bar{z}_{j}^{-2} C\left(\bar{z}_{1}^{-1}, \ldots, \bar{z}_{n}^{-1}\right)\right) \\
& =\sum_{a_{1}, \ldots, a_{n}}\left(\prod_{j} d_{m_{j}, a_{j}} \bar{z}_{j}^{-2-m_{j}-a_{j}}\right) C\left(a_{1}, \bar{z}_{1}^{-1}, \ldots, a_{n}, \bar{z}_{n}^{-1}\right)
\end{aligned}
$$

Here in the last line we have defined integers $d_{m, a}$ by computing for any analytic function $f$

$$
\bar{\partial}^{m-1}\left(-\bar{z}^{-2} \partial f\left(\bar{z}^{-1}\right)\right)=\sum_{a=0}^{m} d_{m, a} \bar{z}^{-2-m-a} \partial^{a} f\left(\bar{z}^{-1}\right)
$$

or equivalently

$$
\bar{\partial}^{m}\left(f\left(\bar{z}^{-1}\right)\right)=\sum_{a=0}^{m} d_{m, a} \bar{z}^{-2-m-a} \partial^{a} f\left(\bar{z}^{-1}\right)
$$

\section{2}

We want to work the derivatives into our basic Hilbert space structure. Let $\Upsilon^{\prime}$ be the free algebra generated by the sequences $Z=\left[m_{1}, z_{1}, \ldots, m_{n}, z_{n}\right]$ with $m_{i} \geq 1$ and $z_{i} \in \mathbb{C}$. We identify $\Upsilon$ as the subalgebra generated by $\left[1, z_{1}, \ldots, 1, z_{n}\right]$. Let $\Upsilon_{0}^{\prime}$ elements of $\Upsilon^{\prime}$ with non-coinciding points. We extend our expectation on $\Upsilon_{0}$ to an expectation on $\Upsilon_{0}^{\prime}$ given by

$$
<Z>=<\left[m_{1}, z_{1}, \ldots, m_{n}, z_{n}\right]>=C\left(m_{1}, z_{1}, \ldots, m_{n}, z_{n}\right)
$$

Thus $\left[m_{1}, z_{1}, \ldots, m_{n}, z_{n}\right]$ stands for the formal $\partial^{m_{1}} X\left(z_{1}\right) \ldots \partial^{m_{n}} X\left(z_{n}\right)$.

For each $a \in \mathbb{C}, q \in \mathbb{C}-\{0\}$ we define an define an extended automorphism $J$ on $\Upsilon^{\prime}$ by

$$
J\left[m_{1}, z_{1}, \ldots, m_{n}, z_{n}\right]=\left[m_{1}, a+q z_{1}, \ldots, m_{n}, a+q z_{n}\right] \prod_{i} q^{m_{i}}
$$

Then by (57) we have $\langle J Z>=<Z>$. by

Taking a cue from (58) we define an extended reflection as an anti-linear automorphism on $\Upsilon_{\mathbb{C}-\{0\}}^{\prime}$

$$
\Theta\left[m_{1}, z_{1}, \ldots, m_{n}, z_{n}\right]=\sum_{a_{1}, \ldots, a_{n}}\left(\prod_{j} d_{m_{j}, a_{j}} \bar{z}^{-2-m_{j}-a_{j}}\right)\left[a_{1}, \bar{z}_{1}^{-1}, \ldots, a_{n}, \bar{z}_{n}^{-1}\right]
$$

Then $\overline{<Z>}=<\Theta Z>$. 
Lemma $3 \quad \Theta^{2}=I$

Proof. This follows from the identity

$$
\sum_{0 \leq b \leq a \leq m} d_{m, a} d_{a, b}=\delta_{m, b}
$$

To see this let $g(z)=f(1 / z)$ and then by definition

$$
\left(\partial^{a} g\right)(z)=\sum_{0 \leq b \leq a} d_{a, b} z^{-2-a-b}\left(\partial^{b} f\right)(1 / z)
$$

On the other hand since $f(z)=g(1 / z)$

$$
\left(\partial^{m} f\right)(z)=\sum_{0 \leq a \leq m} d_{m, a} z^{-2-m-a}\left(\partial^{a} g\right)(1 / z)
$$

Substitute (65) at $1 / z$ into (66) and obtain

$$
\left(\partial^{m} f\right)(z)=\sum_{0 \leq b \leq a \leq m} d_{m, a} d_{a, b} z^{b-m}\left(\partial^{b} f\right)(z)
$$

Now take $f(z)=e^{z}$ and get

$$
z^{m}=\sum_{0 \leq b \leq m}\left(\sum_{b \leq a \leq m} d_{m, a} d_{a, b}\right) z^{b}
$$

Matching coefficients we have the desired result.

Now let $\Upsilon_{0, D-\{0\}}^{\prime}$ be elements with non-coinciding points in $D-\{0\}$ and extend the definition of $\nu_{0}: \Upsilon_{0, D-\{0\}} \mapsto \mathcal{H}$ to a linear map $\nu_{0}: \Upsilon_{0, D-\{0\}}^{\prime} \mapsto \mathcal{H}$ by

$$
\nu_{0}(Z) \equiv \nu_{0}\left(\left[m_{1}, z_{1}, \ldots m_{n}, z_{n}\right]\right)=\partial_{z_{1}}^{m_{1}-1} \ldots \partial_{z_{n}}^{m_{n}-1} \nu_{0}\left(\left[z_{1}, \ldots, z_{n}\right]\right)
$$

Lemma 4 For $F, G \in \Upsilon_{0, D-\{0\}}^{\prime}$

$$
\left(\nu_{0}(F), \nu_{0}(G)\right)=<(\Theta F) G>
$$

Proof. For $Z=\left[m_{1}, z_{1}, \ldots m_{n}, z_{n}\right]$ and $W=\left[\ell_{1}, w_{1}, \ldots, \ell_{r}, w_{r}\right]$

$$
\begin{aligned}
\left.\left(\nu_{0}(Z), \nu_{0}(W)\right)\right) & =\partial_{\bar{z}_{1}}^{m_{1}-1} \cdots \partial_{\bar{z}_{n}}^{m_{n}-1} \partial_{w_{1}}^{\ell_{1}-1} \cdots \partial_{w_{r}}^{\ell_{r}-1}\left(\nu_{0}\left(\left[z_{1}, \ldots, z_{n}\right]\right), \nu_{0}\left(\left[w_{1}, \ldots, w_{r}\right]\right)\right) \\
& =\partial_{\bar{z}_{1}}^{m_{1}-1} \cdots \partial_{\bar{z}_{n}}^{m_{n}-1} \partial_{w_{1}}^{\ell_{1}-1} \cdots \partial_{w_{r}}^{\ell_{r}-1} \prod_{j}\left(-\bar{z}_{j}^{-2}\right)<\left[\bar{z}_{1}^{-1}, \ldots, \bar{z}_{n}^{-1}, w_{1}, \ldots, w_{r}\right]> \\
& =\sum_{a_{1}, \cdots a_{r}}\left(\prod_{j} d_{m_{j}, a_{j}} \bar{z}_{j}^{-2-m_{j}-a_{j}}\right)<\left[a_{1}, \bar{z}_{1}^{-1}, \ldots, a_{n}, \bar{z}_{n}^{-1}, \ell_{1}, w_{1}, \ldots, \ell_{r}, w_{r}\right]> \\
& =<(\Theta Z) W>
\end{aligned}
$$

The general result follows. 


\section{Wick products}

\section{1}

Now we can define Wick products as elements of $\Upsilon$ by

$$
: Z:_{0}=:\left[z_{1}, \ldots, z_{n}\right]:_{0}=\sum_{Q} \prod_{\{\alpha, \beta\} \in Q}\left(-C\left(z_{\alpha}, z_{\beta}\right)\right) \prod_{\gamma \notin Q}\left[z_{\gamma}\right]
$$

where $Q$ is a collection of pairs from $(1, \ldots, n)$ and we must restrict to non-coinciding points. More generally in $\Upsilon^{\prime}$

$$
: Z:_{0}=:\left[m_{1}, z_{1}, \ldots, m_{n}, z_{n}\right]:_{0}=\sum_{Q} \prod_{\{\alpha, \beta\} \in Q}\left(-C\left(m_{\alpha}, z_{\alpha}, m_{\beta}, z_{\beta}\right)\right) \prod_{\gamma \notin Q}\left[m_{\gamma}, z_{\gamma}\right]
$$

where $C$ is defined in (56). If there is a single point then : $[m, z]:_{0}=[m, z]$. For future reference we note that Wick products satisfy the identity

$$
\begin{aligned}
& {[m, z]:\left[m_{1}, z_{1}, \ldots, m_{n}, z_{n}\right]:_{0} } \\
= & :\left[m, z, m_{1}, z_{1}, \ldots, m_{n}, z_{n}\right]:_{0}+\sum_{j=1}^{n} C\left(m, z, m_{j}, z_{j}\right):\left[m_{1}, z_{1}, \ldots, \widehat{m_{j}, z_{j}}, \ldots, m_{n}, z_{n}\right]:_{0}
\end{aligned}
$$

where the hat means omit $m_{j}, z_{j}$.

Now consider several Wick products

$$
: Z^{i}:_{0}=:\left[m_{1}^{i}, z_{1}^{i}, \ldots, m_{n_{i}}^{i} z_{n_{i}}^{i}\right]:_{0}
$$

Products : $Z^{1}:_{0} \cdots: Z^{r}:_{0}$ are elements of $\Upsilon^{\prime}$ and if no points coincide we can consider the expectation $<: Z^{1}:_{0} \cdots: Z^{r}:_{0}>$. As is well known Wick products suppress pairings within the dots. Hence

$$
<: Z^{1}:_{0} \cdots: Z^{r}:_{0}:>=\sum_{P^{\prime}} \prod_{\{(i, \alpha),(j, \beta)\} \in P^{\prime}} C\left(m_{\alpha}^{i}, z_{\alpha}^{i}, m_{\beta}^{j}, z_{\beta}^{j}\right)
$$

where the sum is over pairings $P^{\prime}=\{\{(i, \alpha),(j, \beta)\}\}$ with $i \neq j$. This can be thought of a sum over graphs on $r$ vertices with $n_{i}$ legs at the $i^{t h}$ vertex. The correlation function is invariant under permutation of the $Z$ 's and under permutations within the $Z$ 's.

We collect some properties of Wick products.

\section{Lemma 5}

1. The automorphism $J$ defined by (62) satisfies

$$
J:\left[m_{1}, z_{1}, \ldots, m_{n}, z_{n}\right]:_{0}=:\left[m_{1}, a+q z_{1}, \ldots, m_{n}, a+q z_{n}\right]:_{0} \prod_{i} q^{m_{i}}
$$

2. The anti-linear automorphism defined by (63) satisfies

$$
\Theta:\left[m_{1}, z_{1}, \ldots, m_{n}, z_{n}\right]::_{0}=\sum_{a_{1}, \ldots, a_{n}}\left(\prod_{j} d_{m_{j}, a_{j}} \bar{z}^{-2-m_{j}-a_{j}}\right):\left[a_{1}, \bar{z}_{1}^{-1}, \ldots, a_{n}, \bar{z}_{n}^{-1}\right]: 0
$$

3. $\nu_{0}\left(:\left[z_{1}, \ldots, z_{n}\right]:_{0}\right)$ is analytic for non-coinciding points $z_{i} \in D-\{0\}$ and

$$
\partial_{z_{1}}^{m_{1}-1} \ldots \partial_{z_{n}}^{m_{n}-1} \nu_{0}\left(:\left[z_{1}, \ldots, z_{n}\right]:_{0}\right)=\nu_{0}\left(:\left[m_{1}, z_{1}, \ldots m_{n}, z_{n}\right]:_{0}\right)
$$


Proof. Each of these follows from the corresponding formula without the Wick ordering. We check the second in detail. By definition

$$
\Theta:\left[z_{1}, m_{1}, \ldots, z_{n}, m_{n}\right]::_{0}=\sum_{Q} \prod_{\{\alpha, \beta\} \in Q}\left(-\overline{C\left(m_{\alpha}, z_{\alpha}, m_{\beta}, z_{\beta}\right)}\right) \Theta\left(\prod_{\gamma \notin Q}\left[m_{\gamma}, z_{\gamma}\right]\right)
$$

However

$$
\Theta\left(\prod_{\gamma \notin Q}\left[m_{\gamma}, z_{\gamma}\right]\right)=\sum_{\left\{a_{\gamma}\right\}}\left(\prod_{\gamma \notin Q} d_{m_{\gamma}, a_{\gamma}} \bar{z}^{-2-m_{\gamma}-a_{\gamma}}\right)\left[a_{\gamma}, \bar{z}_{\gamma}^{-1}\right]
$$

and by (28)

$$
\begin{aligned}
\overline{C\left(m_{\alpha}, z_{\alpha}, m_{\beta}, z_{\beta}\right)} & =\partial_{\bar{z}_{\alpha}-1}^{m_{\alpha}-1} \partial_{\bar{z}_{\beta}}^{m_{\beta}-1} \overline{C\left(z_{\alpha}, z_{\beta}\right)} \\
& =\partial_{\bar{z}_{\alpha}}^{m_{\alpha}-1} \partial_{\bar{z}_{\beta}}^{m_{\beta}-1}\left(-\bar{z}_{\alpha}^{-2}\right)\left(-\bar{z}_{\beta}^{-2}\right) C\left(\bar{z}_{\alpha}^{-1}, \bar{z}_{\beta}^{-1}\right) \\
& =\sum_{a_{\alpha}, a_{\beta}} d_{m_{\alpha}, a_{\alpha}} d_{m_{\beta}, a_{\beta}} \bar{z}^{-2-m_{\alpha}-a_{\alpha}} \bar{z}^{-2-m_{\beta}-a_{\beta}} C\left(a_{\alpha}, \bar{z}_{\alpha}^{-1}, a_{\beta}, \bar{z}_{\beta}^{-1}\right)
\end{aligned}
$$

Thus

$$
\begin{aligned}
& \Theta:\left[m_{1}, z_{1}, \ldots, m_{n}, z_{n}\right]: \\
= & \sum_{a_{1}, \ldots, a_{n}}\left(\prod_{j=1}^{n} d_{m_{j}, a_{j}} \bar{z}^{-2-m_{j}-a_{j}}\right)\left[\sum_{Q} \prod_{\{\alpha, \beta\} \in Q}\left(-C\left(a_{\alpha}, \bar{z}_{\alpha}^{-1}, a_{\beta}, \bar{z}_{\beta}^{-1}\right)\right) \prod_{\gamma \notin Q}\left[a_{\gamma}, \bar{z}_{\gamma}^{-1}\right]\right]
\end{aligned}
$$

The bracketed expression is identified as : $\left[a_{1}, \bar{z}_{1}^{-1}, \ldots, a_{n}, \bar{z}_{n}^{-1}\right]:$ to complete the proof.

\section{2}

Next want to extend our results to include coinciding points within the Wick products. The correlation functions are defined for such objects, but they are not in our algebra. So we first enlarge the algebra.

Let $\Upsilon^{w}$ be the free algebra generated by (non-empty) symbols

$$
: Z:=:\left[m_{1}, z_{1}, \ldots m_{n}, z_{n}\right]:
$$

with $m_{i} \geq 1$ and $z_{i} \in \mathbb{C}$. The general element is a linear combination of finite sequences : $Z^{1}: \cdots: Z^{r}$ : (possibly empty) and has the form

$$
F=\sum_{Z^{1}, \ldots Z^{r}} F\left(Z^{1}, \ldots, Z^{r}\right): Z^{1}: \cdots: Z^{r}:
$$

Let $\Upsilon_{0}^{w}$ be the subset with no coinciding points between Wick products; within Wick products points may coincide. For $F \in \Upsilon_{0}^{w}$ we can define an expectation $\langle F\rangle$ by

$$
<: Z^{1}: \cdots: Z^{r}:>=\sum_{P^{\prime}} \prod_{\{(i, \alpha),(j, \beta)\} \in P^{\prime}} C\left(m_{\alpha}^{i}, z_{\alpha}^{i}, m_{\beta}^{j}, z_{\beta}^{j}\right)
$$

This is analytic in $z_{\alpha}^{i} \in \mathbb{C}$ with $z_{\alpha}^{i} \neq z_{\beta}^{j}$ for $i \neq j$.

Let $\Upsilon_{00}^{w}$ be elements of $\Upsilon_{0}^{w}$ in which points within the Wick groups do not coincide, so there are no coinciding points at all. For such elements the expectation agrees with the expectation on $\Upsilon_{0}^{\prime}$, that is

$$
<: Z^{1}: \cdots: Z^{r}:>=<: Z^{1}:_{0} \cdots: Z^{r}:_{0}>
$$

Now consider the homomorphism from $\alpha$ from $\Upsilon_{00}^{w}$ to $\Upsilon_{0}^{\prime}$ defined by $\alpha(: Z:)=: Z:_{0}$ that is

$$
\alpha\left(:\left[m_{1}, z_{1}, \ldots, m_{n}, z_{n}\right]:\right)=:\left[m_{1}, z_{1}, \ldots, m_{n}, z_{n}\right]:_{0}
$$


This is onto since : $\left[m_{1}, z_{1}\right]: \cdots:\left[m_{n}, z_{n}\right]:$ is sent to $\left[m_{1}, z_{1}, \ldots, m_{n}, z_{n}\right]$. It is not one-to-one since for example if $z_{1} \neq z_{2}$ both : $\left[1, z_{1}, 1, z_{2}\right]:$ and : $\left[1, z_{1}\right]::\left[1, z_{2}\right]:-C\left(z_{1}, z_{2}\right)$ are sent to : $\left[1, z_{1}, 1, z_{2}\right]: 0$. By (87) for $F \in \Upsilon_{00}^{w}$

$$
<\alpha(F)>=<F>
$$

We define $J$ on $\Upsilon^{w}$ by the formula (77) and then $\alpha \circ J=J \circ \alpha$. For $F \in \Upsilon_{0}^{w}$ we have $\langle J F\rangle=<F>$ directly from (86). We also define $\Theta$ on $\Upsilon_{\mathbb{C}-\{0\}}^{w}$ by the formula (178). Then $\alpha \circ \Theta=\Theta \circ \alpha$ and $\Theta^{2}=I$. Furthermore define a linear mapping $\nu: \Upsilon_{00, D-\{0\}}^{w} \rightarrow \mathcal{H}$ by $\nu=\nu_{0} \circ \alpha$ and then for $F, G \in \Upsilon_{00, D-\{0\}}^{w}$ we have $(\nu(F), \nu(G))=<\Theta(F) G>$. We note in particular that $\nu$ is defined so

$$
\nu\left(:\left[m_{1}, z_{1}, \ldots, m_{n}, z_{n}\right]:\right)=\nu_{0}\left(:\left[m_{1}, z_{1}, \ldots, m_{n}, z_{n}\right]:_{0}\right)
$$

All this is just a reformulation of what we already had. But now we extend to allow coinciding points within the Wick product.

Lemma 6 The mapping $\nu: \Upsilon_{00, D-\{0\}}^{w} \rightarrow \mathcal{H}$ extends to a linear map $\nu: \Upsilon_{0, D-\{0\}}^{w} \rightarrow \mathcal{H}$ such that for $F, G \in \Upsilon_{0, D-\{0\}}^{w}$

$$
(\nu(F), \nu(G))=<\Theta(F) G>
$$

Proof. Each sequence $Z=:\left[m_{1}, z_{1}, \ldots, m_{n}, z_{n}\right]: \in \Upsilon_{0, D-\{0\}}^{w}$ can be approximated by a a sequence $Z_{k}=:\left[m_{1}, z_{1, k}, \ldots, m_{n}, z_{n, k}\right]: \in \Upsilon_{00, D-\{0\}}^{w}$ with non-coinciding points such that $z_{j, k} \rightarrow z_{j}$ as $k \rightarrow \infty$. Then

$$
\nu\left(: Z^{1}: \cdots: Z^{r}:\right) \equiv \lim _{k \rightarrow \infty} \nu\left(: Z_{k}^{1}: \cdots: Z_{k}^{r}:\right)
$$

exists and is independent of the approximating sequence. This follows from the continuity of the correlation functions. This defines $\nu$ on a basis for $\Upsilon_{0, D-\{0\}}^{w}$ and hence as a linear function on the whole algebra. In the same way any $F \in \Upsilon_{0, D-\{0\}}^{w}$ can be approximated by a sequence $F_{k} \in$ $\Upsilon_{00, D-\{0\}}^{w}$ such that $\nu(F)=\lim _{k \rightarrow \infty} \nu\left(F_{k}\right)$. Taking the limit of $\left(\nu\left(F_{k}\right), \nu\left(G_{k}\right)\right)=<\Theta\left(F_{k}\right) G_{k}>$ we get $(\nu(F), \nu(G))=<\Theta(F) G>$.

\section{3}

Next we would like to include the point $z=0$ in our analysis, that is work with $\Upsilon_{0, D}^{w}$ rather than $\Upsilon_{0, D-\{0\}}^{w}$. First we have

Lemma $7 \quad$ Let $z_{i}, w_{j} \in D-\{0\}$. Then

$$
\begin{aligned}
& \left(\nu\left(:\left[m_{1}, z_{1}, \ldots m_{n}, z_{n}\right]:\right), \nu\left(:\left[\ell_{1}, w_{1}, \ldots, \ell_{r}, w_{r}\right]:\right)\right) \\
= & \partial_{\bar{z}_{1}}^{m_{1}-1} \cdots \partial_{\bar{z}_{n}}^{m_{n}-1} \partial_{w_{1}}^{\ell_{1}-1} \ldots \partial_{w_{r}}^{\ell_{r}-1}\left(\sum_{P^{\prime}} \prod_{\{\alpha, \beta\} \in P^{\prime}}\left(1-\bar{z}_{\alpha} w_{\beta}\right)^{-2}\right)
\end{aligned}
$$

where the sum is over pairings $P^{\prime}$ of $(1, \ldots, n)$ with $(1, \ldots, r)$ (empty if $\left.r \neq n\right)$. 
Proof. If all the $m_{i}=1$ we compute

$$
\begin{aligned}
\left(\nu\left(:\left[z_{1}, \ldots, z_{n}\right]:\right), \nu\left(:\left[w_{1}, \ldots, w_{r}\right]:\right)\right) & =<\left(\Theta:\left[z_{1}, \ldots, z_{n}\right]:\right):\left[w_{1}, \ldots, w_{r}\right]:> \\
& =\prod_{i} \frac{-1}{\bar{z}_{i}^{2}}\left\langle: \bar{z}_{1}^{-1}, \ldots, \bar{z}_{n}^{-1}:: w_{1}, \ldots, w_{r}:\right\rangle \\
& =\prod_{i} \frac{-1}{\bar{z}_{i}^{2}} \sum_{P^{\prime}} \prod_{\{\alpha, \beta\} \in P^{\prime}} C\left(\bar{z}_{\alpha}^{-1}, w_{\beta}\right) \\
& =\sum_{P^{\prime}} \prod_{\{\alpha, \beta\} \in P^{\prime}} \frac{1}{2}\left(1-\bar{z}_{\alpha} w_{\beta}\right)^{-2}
\end{aligned}
$$

The result now follows since by (79)

$$
\begin{aligned}
& \left(\nu\left(:\left[m_{1}, z_{1}, \ldots m_{n}, z_{n}\right]:\right), \nu\left(:\left[\ell_{1}, w_{1}, \ldots, \ell_{r}, w_{r}\right]:\right)\right) \\
= & \partial_{\bar{z}_{1}}^{m_{1}-1} \cdots \partial_{\bar{z}_{n}}^{m_{n}-1} \partial_{w_{1}}^{\ell_{1}-1} \cdots \partial_{w_{r}}^{\ell_{r}-1}\left(\nu\left(:\left[z_{1}, \ldots, z_{n}\right]:\right), \nu\left(:\left[w_{1}, \ldots, w_{r}\right]:\right)\right)
\end{aligned}
$$

Lemma 8 The mapping $\nu: \Upsilon_{0, D-\{0\}}^{w} \rightarrow \mathcal{H}$ extends to a linear map $\nu: \Upsilon_{0, D}^{w} \rightarrow \mathcal{H}$ such that for $F \in \Upsilon_{0, D}^{w}$ and $G \in \Upsilon_{0, D-\{0\}}^{w}$

$$
(\nu(G), \nu(F))=<\Theta(G) F>
$$

Proof. Each sequence : $Z:=:\left[m_{1}, z_{1}, \ldots, m_{n}, z_{n}\right]:$ with $z_{i} \in D$ can be approximated by a a sequence $: Z_{k}:=:\left[m_{1}, z_{1, k}, \ldots, m_{n}, z_{n, k}\right]:$ with $z_{i, k} \in D-\{0\}$. Then $\nu(: Z:) \equiv \lim _{k \rightarrow \infty} \nu\left(: Z_{k}:\right)$ exists and is independent of the approximating sequence. This follows from the representation (93).

More generally suppose $: Z^{1}:: Z^{2}: \cdots: Z^{r}: \in \Upsilon_{0, D}^{w}$ has zeros in one factor, say $: Z^{1}:$. This can be approximated by $: Z_{k}^{1}:: Z^{2}: \cdots: Z^{r}: \in \Upsilon_{0, D-\{0\}}^{w}$ and then

$$
\nu\left(: Z^{1}:: Z^{2}: \cdots: Z^{r}:\right) \equiv \lim _{k \rightarrow \infty} \nu\left(: Z_{k}^{1}:: Z^{2}: \cdots: Z^{r}:\right)
$$

exists and is independent of the approximating sequence. This defines $\nu$ on a basis for $\Upsilon_{0, D}^{w}$ and hence as a linear function on the whole algebra. In the same way any $F \in \Upsilon_{0, D}^{w}$ can be approximated by a sequence $F_{k} \in \Upsilon_{0, D-\{0\}}^{w}$ such that $\nu(F)=\lim _{k \rightarrow \infty} \nu\left(F_{k}\right)$. Then $(\nu(G), \nu(F))=<\Theta(G) F>$ follows by taking the limit of $\left(\nu(G), \nu\left(F_{k}\right)\right)=<\Theta(G) F_{k}>$.

Corollary 2 For Wick products : $Z^{i}:=:\left[m_{1}^{i} z_{1}^{i} \ldots, m_{n_{i}}^{i} z_{n_{i}}^{i}\right]:$ the function $\nu\left(: Z^{1}: \cdots: Z^{r}:\right)$ is strongly analytic in $z_{\alpha}^{i} \in D, z_{\alpha}^{i} \neq z_{\beta}^{j}$ for $i \neq j$. It is also symmetric under permutation of the $: Z^{i}$ : and under permutations within the $: Z^{i}:$.

Proof. As in corollary $1\left(\nu(F), \nu\left(: Z^{1}: \cdots: Z^{r}:\right)\right)$ is analytic for all $F \in \Upsilon_{0, D-\{0\}}^{w}$. Such $\nu(F)$ form a dense set, hence $\nu\left(: Z^{1}: \cdots: Z^{r}:\right)$ is weakly analytic, and hence strongly analytic. The symmetry follows similarly. 


\section{Creation and annihilation operators}

Next we define creation and annihilation operators.

Theorem 3 For $m \in \mathbb{Z}$ there exist operators $\alpha_{m}$ defined on $\nu\left(\Upsilon_{0, D-\{0\}}^{w}\right)$ such that

$$
\alpha_{m} \nu(F)=\sqrt{2} \oint_{|z|=r} \frac{d z}{2 \pi} z^{m} \nu([1, z] F)
$$

with $0<r<1$ selected to enclose all the points in $F$. (By the analyticity the expression is independent of the choice of $r$.) Furthermore with $\Omega=\nu(\emptyset)$

1. $\left(\alpha_{m}\right)^{*}=\alpha_{-m}$

2. $\alpha_{m} \Omega=0$ for $m \geq 0$.

3. $\left[\alpha_{m}, \alpha_{n}\right]=m \delta_{m+n}$

Remark. These are standard arguments which we adapt to our setup.

Proof. Abbreviating $[1, z]$ as $[z]$ we first define $\alpha_{m}$ on $F \in \Upsilon_{0, D-\{0\}}^{w}$ by

$$
\alpha_{m} F=\sqrt{2} \oint_{|z|=r} \frac{d z}{2 \pi} z^{m} \nu([z] F)
$$

Then making the change of variables $z=\bar{w}^{-1}, d z=-\bar{w}^{-2} d \bar{w}$ we have

$$
\begin{aligned}
\left(\nu(G), \alpha_{m} F\right) & =\sqrt{2} \oint_{|z|=r} \frac{d z}{2 \pi} z^{m}(\nu(G), \nu([z] F)) \\
& =\sqrt{2} \oint_{|z|=1} \frac{d z}{2 \pi} z^{m}<(\Theta G)[z] F> \\
& =\sqrt{2} \oint_{|w|=1} \frac{d \bar{w}}{2 \pi}\left(-\bar{w}^{-m-2}\right)<(\Theta G)\left[\bar{w}^{-1}\right] F> \\
& =\sqrt{2} \oint_{|w|=1} \frac{d \bar{w}}{2 \pi} \bar{w}^{-m}<(\Theta[w] G) F> \\
& =\sqrt{2} \oint_{|w|=r} \frac{d \bar{w}}{2 \pi} \bar{w}^{-m}(\nu([w] G), \nu(F)) \\
& =\left(\alpha_{-m} \nu(G), \nu(F)\right)
\end{aligned}
$$

Now if $\nu(F)=0$ then $\left(\nu(G), \alpha_{m} F\right)=0$ for all $\nu(G)$ and hence $\alpha_{m} F=0$. Thus we can define $\alpha_{m}$ on $\nu\left(\Upsilon_{0, D-\{0\}}^{w}\right)$ by $\alpha_{m} \nu(F)=\alpha_{m} F$. Then (98) holds and (100) can be written

$$
\left(\nu(G), \alpha_{m} \nu(F)=\left(\alpha_{-m} \nu(G), \nu(F)\right)\right.
$$

which says $\left(\alpha_{m}\right)^{*}=\alpha_{-m}$.

The result $\alpha_{m} \Omega=0$ for $m \geq 0$ follows by analyticity.

For the third item we compute

$$
\left(\nu(G),\left[\alpha_{m}, \alpha_{n}\right] \nu(F)\right)=2 \int_{\Gamma^{+}-\Gamma_{-}} \frac{d z}{2 \pi} \oint_{|\zeta|=r} \frac{d \zeta}{2 \pi} z^{m} \zeta^{n}<(\Theta G)[z, \zeta] F>
$$


where $\Gamma^{ \pm}$are the contours $|z|=r \pm \epsilon$ and $\epsilon$ is sufficiently small. Now we claim that

$$
<(\Theta G)[z, \zeta] F>=C(z, \zeta)<(\Theta G) F>+\cdots
$$

where the term $\ldots$ is analytic in $z$ between $\Gamma^{ \pm}$. To see this recall that the correlation functions are written as sums over pairings. If the points $z, \zeta$ are not paired with each other then they are paired with something outside of the corridor between $\Gamma^{ \pm}$and hence the expression is analytic between $\Gamma^{ \pm}$. The terms where they are paired with each other have the claimed form. In the integral the analytic term contribute nothing. For the other term we compute

$$
\oint_{|\zeta|=r} \frac{d \zeta}{2 \pi} \int_{\Gamma^{+}-\Gamma^{-}} \frac{d z}{2 \pi} \frac{-z^{m} \zeta^{n}}{(z-\zeta)^{2}}=\oint_{|\zeta|=r} \frac{d \zeta}{2 \pi}(-i m) \zeta^{n+m-1}=m \delta_{m+n}
$$

Thus we get

$$
\left(\nu(G),\left[\alpha_{m}, \alpha_{n}\right] \nu(F)\right)=m \delta_{m+n}(\nu(G), \nu(F))
$$

as required. This completes the proof.

Now consider vectors $\nu\left(:\left[z_{1}, \ldots, z_{n}\right]:\right) \equiv \nu\left(:\left[1, z_{1}, \ldots, 1, z_{n}\right]:\right)$. By Corollary 2 this is analytic in $z_{i} \in D$ and hence has a convergent power series in this region. The next result identifies the coefficients in the series

Lemma 9 For $z_{i} \in D$

$$
\nu\left(:\left[z_{1}, \ldots, z_{n}\right]:\right)=\left(\frac{1}{\sqrt{2} i}\right)^{n / 2} \sum_{m_{1}, \ldots, m_{n} \geq 0} z_{1}^{m_{1}} \ldots z_{n}^{m_{n}}\left(\alpha_{-m_{1}-1} \cdots \alpha_{-m_{n}-1} \Omega\right)
$$

or

$$
\nu\left(:\left[z_{1}, \ldots, z_{n}\right]:\right)=\left(\frac{1}{\sqrt{2} i}\right)^{n / 2} \sum_{m_{1}, \ldots, m_{n} \geq 1} z_{1}^{m_{1}-1} \ldots z_{n}^{m_{n}-1}\left(\alpha_{-m_{1}} \cdots \alpha_{-m_{n}} \Omega\right)
$$

Proof. The coefficient of $z_{1}^{m_{1}} \ldots z_{n}^{m_{n}}$ is

$$
\int_{\left|z_{n}\right|=r_{n}} \frac{d z_{n}}{2 \pi i} \frac{1}{z_{n}^{m_{n}+1}} \cdots \int_{\left|z_{1}\right|=r_{1}} \frac{d z_{1}}{2 \pi i} \frac{1}{z_{1}^{m_{1}+1}} \nu\left(:\left[z_{1}, \ldots, z_{n}\right]:\right)
$$

for any $0<r_{i}<1$ and we take $r_{1}<r_{2}<\cdots<r_{n}$. But for distinct $z_{i}$

$$
\nu\left(:\left[z_{1}, \ldots, z_{n}\right]:\right)=\nu\left(\left[z_{1}\right]:\left[z_{2}, \ldots, z_{n}\right]:\right)-\sum_{j=1}^{n} C\left(z_{1}, z_{j}\right) \nu\left(:\left[z_{1}, \ldots, \widehat{z_{j}}, \ldots, z_{n}\right]:\right)
$$

This is true with $\nu_{0}\left(:\left[z_{1}, \ldots, z_{n}\right]:_{0}\right)$ by (74) and hence also holds as stated by (90). The second term is analytic inside $\left|z_{1}\right|=r_{1}$ and so does not contribute to the integral. For the first term we have

$$
\int_{\left|z_{1}\right|=r_{1}} \frac{d z_{1}}{2 \pi i} \frac{1}{z_{1}^{m_{1}+1}} \nu\left(\left[z_{1}\right]:\left[z_{2}, \ldots, z_{n}\right]:\right)=\frac{1}{\sqrt{2} i} \alpha_{-m_{1}-1} \nu\left(:\left[z_{2}, \ldots, z_{n}\right]:\right)
$$

Repeating this argument 1 we find the coefficient of $z_{1}^{m_{1}} \ldots z_{n}^{m_{n}}$ is

$$
\left(\frac{1}{\sqrt{2} i}\right)^{n} \alpha_{-m_{1}-1} \cdots \alpha_{-m_{n}-1} \Omega
$$

\footnotetext{
${ }^{1}$ We are taking the operators $\alpha_{m}$ outside the integral without having established continuity. It is allowed since it suffices to establish the identity weakly
} 
Theorem 4 The vectors $\alpha_{-m_{1}} \cdots \alpha_{-m_{n}} \Omega$ with $m_{i} \geq 1$ span a subspace dense in $\mathcal{H}$. Hence $\mathcal{H}$ is separable and naturally isomorphic to Fock space.

Proof. Vectors of the form $\nu_{0}\left(\left[z_{1}, \ldots, z_{n}\right]\right)$ with $z_{i} \neq z_{j}$ span a dense subspace by construction. But $\left[z_{1}, \ldots, z_{n}\right]$ is a combination of the : $\left[z_{1}, \ldots, z_{n}\right]:_{0}$ (use the identity (744)). Hence the $\nu_{0}\left(:\left[z_{1}, \ldots, z_{n}\right]:_{0}\right.$ ) span a dense subspace and these are the same as the $\nu\left(:\left[z_{1}, \ldots, z_{n}\right]:\right)$. Thus it suffices to approximate vectors $\nu\left(:\left[z_{1}, \ldots, z_{n}\right]:\right)$ with vectors $\alpha_{-m_{1}} \cdots \alpha_{-m_{n}} \Omega$. This follows by truncating the power series (107).

Remark. We note that $\alpha_{0}=0$. This follows on vectors $\alpha_{-m_{1}} \cdots \alpha_{-m_{n}} \Omega$ by $\left[\alpha_{0}, \alpha_{-m}\right]=0$ and $\alpha_{0} \Omega=0$.

\section{Lemma 10}

$$
\alpha_{-m_{1}} \cdots \alpha_{-m_{n}} \Omega=\prod_{j}\left(\frac{\sqrt{2} i}{\left(m_{j}-1\right) !}\right) \quad \nu\left(:\left[m_{1}, 0, \ldots, m_{n}, 0\right]:\right)
$$

or if $n_{m}$ is the number of times $m$ occurs in $m_{1}, \ldots, m_{n}$

$$
\alpha_{-m_{1}} \cdots \alpha_{-m_{n}} \Omega=\prod_{m}\left(\frac{\sqrt{2} i}{(m-1) !}\right)^{n_{m}} \nu\left(: \prod_{m}[m, 0]^{n_{m}}:\right)
$$

Proof. We compute by (79) and (107)

$$
\begin{aligned}
\nu\left(:\left[m_{1}, 0, \ldots, m_{n}, 0\right]:\right) & =\left.\partial_{z_{1}}^{m_{1}-1} \cdots \partial_{z_{n}}^{m_{n}-1} \nu\left(:\left[z_{1}, \ldots, z_{n}\right]:\right)\right|_{z_{i}=0} \\
& =\prod_{j}\left(\frac{\left(m_{j}-1\right) !}{\sqrt{2} i}\right) \alpha_{-m_{1}} \cdots \alpha_{-m_{n}} \Omega
\end{aligned}
$$

Summary: We have constructed a Hilbert space $\mathcal{H}$ from the correlation functions for the model. In this space we have constructed a vacuum $\Omega$ and creation and annihilation operators $\alpha_{m}$ such that vectors $\alpha_{-m_{1}} \cdots \alpha_{-m_{n}} \Omega$ span a dense set, so $\mathcal{H}$ is identified as a Fock space. Furthermore vectors $\alpha_{-m_{1}} \cdots \alpha_{-m_{n}} \Omega$ are identified with states $\nu\left(:\left[m_{1}, 0, \ldots, m_{n}, 0\right]:\right)$ representing Wick products of fields at the origin. These are the basic facts which we need to attack the main problem.

\section{Transition amplitudes}

Now we define transition amplitudes. Let $D_{1}, \ldots, D_{r}$ be a number of disjoint discs in $\mathbb{C}$. They are of the form

$$
D_{i}=\left\{z \in \mathbb{C}:\left|z-a_{i}\right|<R_{i}\right\}
$$

A natural problem is to consider a correlation function of the form $<G_{1} \cdots G_{r}>$ with $G_{i} \in \Upsilon_{D_{i}-\left\{a_{i}\right\}}$ and show that it defines a bounded multi-linear functional on $\mathcal{H}_{D_{1}} \times \cdots \times \mathcal{H}_{D_{n}}$ where $\mathcal{H}_{D}$ is defined in (50). That is we would like to show that it depends only on the equivalence classes $\tilde{\nu}\left(G_{i}\right) \in \mathcal{H}_{D_{i}}$ and that there is a constant $C$ such that

$$
\left|<G_{1} \cdots G_{r}>\right| \leq C\left\|\tilde{\nu}\left(G_{1}\right)\right\|_{D_{1}} \cdots\left\|\tilde{\nu}\left(G_{r}\right)\right\|_{D_{r}}
$$

Then it extends by continuity to $\mathcal{H}_{D_{1}} \times \cdots \times \mathcal{H}_{D_{n}}$. 
It is desirable to refer everything to the standard Hilbert space $\mathcal{H}$ on the disc $D=\{z \in \mathbb{C}:|z|<1\}$. Accordingly we assume the $D_{i}$ are parametrized by mappings $j_{i}$ from $D$ to $D_{i}$ of the form $j_{i}(z)=$ $a_{i}+q_{i} z$ with $\left|q_{i}\right|=R_{i}$. As we have seen in section 2.5 these induce isomorphisms $J_{i}$ from $\Upsilon_{0, D-\{0\}}$ to $\Upsilon_{0, D_{i}-\left\{a_{i}\right\}}$ and unitary maps $\mathcal{J}_{i}$ from $\mathcal{H}$ to $\mathcal{H}_{D_{i}}$ such that $\mathcal{J}_{i} \nu_{0}(F)=\tilde{\nu}\left(J_{i} F\right)$. The problem is reformulated as follows. Given $F_{1}, \ldots, F_{r}$ in $\Upsilon_{0, D-\{0\}}$ show that $\left\langle\left(J_{1} F_{1}\right) \cdots\left(J_{r} F_{r}\right)\right\rangle$ depends only on $\nu_{0}\left(F_{i}\right) \in \mathcal{H}$ and that

$$
\left|<\left(J_{1} F_{1}\right) \cdots\left(J_{r} F_{r}\right)>\right| \leq C\left\|\nu_{0}\left(F_{1}\right)\right\| \cdots\left\|\nu_{0}\left(F_{r}\right)\right\|
$$

If we put $F_{i}=J_{i}^{-1} G_{i}$ we get the previous version.

We further generalize by allowing Wick products. If $F_{1}, \ldots, F_{r}$ in $\Upsilon_{0, D}^{w}$ then $J_{i} F_{i} \in \Upsilon_{0, D_{i}}^{w}$ and we consider correlation functions $\left\langle\left(J_{1} F_{1}\right) \cdots\left(J_{r} F_{r}\right)>\right.$. We seek to show that these depend only on $\nu\left(F_{i}\right) \in \mathcal{H}$ and that

$$
\left|<\left(J_{1} F_{1}\right) \cdots\left(J_{r} F_{r}\right)>\right| \leq C\left\|\nu\left(F_{1}\right)\right\| \cdots\left\|\nu\left(F_{r}\right)\right\|
$$

Given $F_{i}^{\prime} \in \Upsilon_{0, D-\{0\}}$ one can choose $F_{i} \in \Upsilon_{00, D-\{0\}}^{w}$ such that $\alpha\left(F_{i}\right)=F_{i}^{\prime}$ and hence recover the previous version.

Let $\mathcal{D}$ be the dense domain $\mathcal{D} \equiv \nu\left(\Upsilon_{0, D}^{w}\right)$. As a first step we have as in [2]:

Lemma 11 For $F_{i} \in \Upsilon_{0, D}^{w}$ the correlation function $\left\langle J_{1} F_{1}, \ldots, J_{r} F_{r}>\right.$ only depends on $\mathcal{F}_{i}=\nu\left(F_{i}\right) \in$ $\mathcal{D}$. It thus defines a multilinear functional $\mathcal{A}_{r}$ on $\mathcal{D} \times \cdots \times \mathcal{D}$ such that

$$
\mathcal{A}_{r}\left(\mathcal{F}_{1}, \cdots, \mathcal{F}_{r}\right)=<\left(J_{1} F_{1}\right) \ldots\left(J_{r} F_{r}\right)>
$$

Furthermore if $\phi_{\alpha}=\nu\left(\psi_{\alpha}\right) \in \mathcal{D}$ is an orthonormal basis for $\mathcal{H}$ then

$$
\mathcal{A}_{r}\left(\mathcal{F}_{1}, \cdots, \mathcal{F}_{r}\right)=\sum_{\alpha_{1}} \cdots \sum_{\alpha_{r}} \mathcal{A}_{r}\left(\phi_{\alpha_{1}}, \cdots, \phi_{\alpha_{r}}\right)\left(\phi_{\alpha_{r}}, \mathcal{F}_{r}\right) \cdots\left(\phi_{\alpha_{1}}, \mathcal{F}_{1}\right)
$$

Remark. We do not yet assert that the functional is bounded. We also do not yet assert that the multiple sum is absolutely convergent, only that the iterated sum converges.

Proof. Since $\langle J F>=<F>$ we have

$$
<\left(J_{1} F_{1}\right)\left(J_{2} F_{2}\right) \ldots\left(J_{r} F_{r}\right)>=<F_{1}\left(J_{1}^{-1} J_{2} F_{2}\right) \ldots\left(J_{1}^{-1} J_{r} F_{r}\right)>
$$

Since the $D_{i}$ are disjoint we have for $i \neq 1$ that $j_{i} D=D_{i} \subset D_{1}^{\prime}$, hence $j_{1}^{-1} j_{i} D \subset D^{\prime}$, and hence $J_{1}^{-1} J_{i} F_{i} \in \Upsilon_{0, D^{\prime}}^{w}$. Since $\Theta^{2}=I$ it follows that $\left(J_{1}^{-1} J_{2} F_{2}\right) \ldots\left(J_{1}^{-1} J_{n} F_{n}\right)$ has the form $\Theta F$ for some $F \in \Upsilon_{0, D-\{0\}}^{w}$ and so by (96)

$$
<\left(J_{1} F_{1}\right)\left(J_{2} F_{2}\right) \ldots\left(J_{r} F_{r}\right)>=<(\Theta F) F_{1}>=\left(\nu(F), \nu\left(F_{1}\right)\right)
$$

Thus the expression only depends on $\mathcal{F}_{1}=\nu\left(F_{1}\right)$. A similar argument establishes the result in the other variables and hence the first result.

To get the expansion we compute from (122)

$$
\begin{aligned}
\mathcal{A}_{r}\left(\mathcal{F}_{1}, \ldots, \mathcal{F}_{r}\right) & =\sum_{\alpha_{1}}\left(\nu(F), \phi_{\alpha_{1}}\right)\left(\phi_{\alpha_{1}}, \nu\left(F_{1}\right)\right) \\
& =\sum_{\alpha_{1}}<\Theta(F) \psi_{\alpha_{1}}>\left(\phi_{\alpha_{1}}, \nu\left(F_{1}\right)\right) \\
& =\sum_{\alpha_{1}}<\left(J_{1} \psi_{\alpha_{1}}\right)\left(J_{2} F_{2}\right) \ldots\left(J_{r} F_{r}\right)>\left(\phi_{\alpha_{1}}, \mathcal{F}_{1}\right) \\
& =\sum_{\alpha_{1}} \mathcal{A}_{r}\left(\phi_{\alpha_{1}}, \mathcal{F}_{2}, \ldots, \mathcal{F}_{r}\right)\left(\phi_{\alpha_{1}}, \mathcal{F}_{1}\right)
\end{aligned}
$$


Repeating this in the other variables gives the result.

Now the question is whether $\mathcal{A}_{r}$ extends from a functional on the dense domain $\mathcal{D} \times \cdots \times \mathcal{D}$ to the full Hilbert space $\mathcal{H} \times \cdots \times \mathcal{H}$. We prove the stronger result that it is a Hilbert-Schmidt functional, i.e. that there is a orthonormal basis $\left\{\phi_{\alpha}\right\}$ for $\mathcal{H}$ with $\phi_{\alpha} \in \mathcal{D}$ such that

$$
\left\|\mathcal{A}_{r}\right\|_{H S}^{2}=\sum_{\alpha_{1}, \ldots, \alpha_{r}}\left|\mathcal{A}_{r}\left(\phi_{\alpha_{1}}, \cdots \phi_{\alpha_{r}}\right)\right|^{2}<\infty
$$

If it converges for one orthonormal basis then it converges for all such bases. Then by the Schwarz inequality the sum (120) is absolutely convergent and we have the bound for $\mathcal{F}_{i} \in \mathcal{D}$

$$
\left|\mathcal{A}_{r}\left(\mathcal{F}_{1}, \ldots, \mathcal{F}_{r}\right)\right| \leq\left\|\mathcal{A}_{r}\right\|_{H S}\|\| \mathcal{F}\left\|_{1} \cdots\right\| \mathcal{F} \|_{n}
$$

Hence $\mathcal{A}_{r}$ extends to a bounded multilinear functional on $\mathcal{H} \times \cdots \times \mathcal{H}$ which is still Hilbert-Schmidt. To state the result precisely define

$$
R=\sup _{i} R_{i} \quad d=\inf _{i, j}\left|a_{i}-a_{j}\right|
$$

We assume that $d / R$ is not too small. Note that the Euclidean distance between $D_{i}$ and $D_{j}$ is

$$
d\left(D_{i}, D_{j}\right)=\left|a_{i}-a_{j}\right|-R_{i}-R_{j} \geq d-2 R
$$

Hence $d / R>2$ guarantees that the discs are separated.

Theorem 5 Let $d / R>4 \sqrt{r} \geq 4$. Then $\mathcal{A}_{r}$ on $\mathcal{D} \times \cdots \times \mathcal{D}$ is a Hilbert-Schmidt functional and so extends to a bounded multilinear functional on $\mathcal{H} \times \cdots \times \mathcal{H}$ satisfying (125).

Proof. An orthonormal basis $\phi\left(\left\{n_{m}\right\}\right)$ for Fock space is indexed by sequences $\left\{n_{m}\right\}=\left\{n_{1}, n_{2}, \ldots\right\}$ which are eventually zero. It has the form

$$
\phi\left(\left\{n_{m}\right\}\right)=\prod_{m}\left(n_{m} ! m^{n_{m}}\right)^{-1 / 2} \prod_{m} \alpha_{-m}^{n_{m}} \Omega
$$

Therefore by (113) an orthonormal basis for $\mathcal{H}$ is

$$
\phi\left(\left\{n_{m}\right\}\right)=\prod_{m}\left(n_{m} !\right)^{-1 / 2}\left(\frac{i \sqrt{2 m}}{m !}\right)^{n_{m}} \nu\left(:\left[m_{1}, 0, \ldots, m_{n}, 0\right]:\right)
$$

Here $\nu\left(:\left[m_{1}, 0, \ldots, m_{n}, 0\right]:\right)$ has $n^{m} m^{\prime}$ s in any order and can also be written $\nu\left(: \prod_{m}[m, 0]^{n_{m}}:\right)$. We also define in $\Upsilon_{0, D}^{w}$

$$
\psi\left(\left\{n_{m}\right\}, z\right)=\prod_{m}\left(n_{m} !\right)^{-1 / 2}\left(\frac{i \sqrt{2 m}}{m !}\right)^{n_{m}}:\left[m_{1}, z, \ldots, m_{n}, z\right]:
$$

and then $\phi\left(\left\{n_{m}\right\}\right)=\nu\left(\psi\left(\left\{n_{m}\right\}, 0\right)\right)$

We want to study

$$
\mathcal{A}_{r}\left(\phi\left(\left\{n_{m}^{1}\right\}\right), \ldots, \phi\left(\left\{n_{m}^{r}\right\}\right)\right)=\left\langle J_{1} \psi\left(\left\{n_{m}^{1}\right\}, 0\right) \cdots J_{r} \psi\left(\left\{n_{m}^{r}\right\}, 0\right)\right\rangle
$$

Since

$$
J_{j} \psi\left(\left\{n_{m}^{j}\right\}, 0\right)=\prod_{m} q_{j}^{n_{m}^{j} m} \psi\left(\left\{n_{m}^{j}\right\}, a_{i}\right)
$$


we have

$$
\mathcal{A}_{r}\left(\phi\left(\left\{n_{m}^{1}\right\}\right), \ldots, \phi\left(\left\{n_{m}^{r}\right\}\right)\right)=\left\langle\psi\left(\left\{n_{m}^{1}\right\}, a_{1}\right) \cdots \psi\left(\left\{n_{m}^{r}\right\}, a_{r}\right)\right\rangle \prod_{j=1}^{r} \prod_{m=1}^{\infty} q_{j}^{n_{m}^{j} m}
$$

By (86):

$$
\begin{aligned}
& \left\langle\psi\left(\left\{n_{m}^{1}\right\}, a_{1}\right) \cdots \psi\left(\left\{n_{m}^{r}\right\}, a_{r}\right)\right\rangle \\
= & \prod_{j, m}\left(n_{m}^{j} !\right)^{-1 / 2}\left(\frac{i \sqrt{2 m}}{m !}\right)^{n_{m}^{j}} \sum_{P^{\prime}} \prod_{\{(i, \alpha),(j, \beta)\} \in P^{\prime}} C\left(m_{\alpha}^{i}, a_{i}, m_{\beta}^{j}, a_{j}\right)
\end{aligned}
$$

Now we estimate these quantities. Using $(a+b) ! \leq a ! b ! 2^{a+b}$ and (56)

$$
\left|C\left(m_{\alpha}^{i}, a_{i}, m_{\beta}^{j}, a_{j}\right)\right| \leq \frac{1}{2}\left(m_{\alpha}^{i}+m_{\beta}^{j}-1\right) ! d^{-m_{\alpha}^{i}-m_{\beta}^{j}} \leq \frac{1}{2^{2}} m_{\alpha}^{i} ! m_{\beta}^{j} !\left(\frac{2}{d}\right)^{m_{\alpha}^{i}+m_{\beta}^{j}}
$$

The $m$ !'s contribute a factor $\prod_{j, \beta} m_{\beta}^{j} !=\prod_{j, m}(m !)^{n_{m}^{j}}$ which is canceled by a similar factor in (134). The factors $(2 / d)^{m_{\alpha}^{i}+m_{\beta}^{j}}$ combine to give an overall $\prod_{j, \beta}(2 / d)^{m_{\beta}^{j}}=\prod_{j, m}(2 / d)^{n_{m}^{j} m}$. The factors $2^{-2}$ contribute a factor $2^{-n}$ where

$$
n=\sum_{j=1}^{r} \sum_{m=1}^{\infty} n_{m}^{j}
$$

is the total number of fields. Also we estimate the number of pairings or graphs by ignoring the restriction that a line cannot join the same vertex. Thus it is fewer than the number of graphs on $n$ legs which is

$$
\frac{n !}{(n / 2) ! 2^{n}} \leq \sqrt{n !} 2^{-n / 2}
$$

and the $2^{-n / 2}$ is canceled by a similar factor in (134). Altogether then we have

$$
\left|\left\langle\psi\left(\left\{n_{m}^{1}\right\}, a_{1}\right) \cdots \psi\left(\left\{n_{m}^{r}\right\}, a_{r}\right)\right\rangle\right| \leq \sqrt{n !} 2^{-n} \prod_{j, m}\left(n_{m}^{j} !\right)^{-1 / 2} m^{n_{m}^{j} / 2}\left(\frac{2}{d}\right)^{n_{m}^{j} m}
$$

We take $m<2^{m}$ in this and combine it with the estimate

$$
\prod_{j, m}\left|q_{j}\right|^{n_{m}^{j} m} \leq \prod_{j, m} R^{n_{m}^{j} m}
$$

to obtain

$$
\left|\mathcal{A}_{r}\left(\phi\left(\left\{n_{m}^{1}\right\}\right), \ldots, \phi\left(\left\{n_{m}^{r}\right\}\right)\right)\right| \leq \sqrt{n !} 2^{-n} \prod_{j, m}\left(n_{m}^{j} !\right)^{-1 / 2}\left(\frac{2^{3 / 2} R}{d}\right)^{n_{m}^{j} m}
$$

We want to sum $\left|\mathcal{A}_{r}\right|^{2}$ over all integers $n_{m}^{j} \geq 0$ with $1 \leq j \leq r$ and $1 \leq m$. We first consider a smaller sum with the additional restriction $m \leq M$. Then by the multinomial expansion: 


$$
\begin{aligned}
\sum_{\left\{\left\{n_{m}^{j}\right\}: m \leq M\right\}}\left|\mathcal{A}_{r}\right|^{2} & \leq \sum_{\left\{\left\{n_{m}^{j}\right\}: m \leq M\right\}} n ! 4^{-n} \prod_{j, m}\left(n_{m}^{j} !\right)^{-1}\left(\frac{8 R^{2}}{d^{2}}\right)^{n_{m}^{j} m} \\
& =\sum_{n=0}^{\infty} 4^{-n} \sum_{\left\{\left\{n_{m}^{j}\right\}: m \leq M, \sum n_{m}^{j}=n\right\}} \frac{n !}{\prod_{j, m} n_{m}^{j} !}\left[\left(\frac{8 R^{2}}{d^{2}}\right)^{m}\right]^{n_{m}^{j}} \\
& =\sum_{n=0}^{\infty} 4^{-n}\left(\sum_{j=1}^{r} \sum_{m=1}^{M}\left(\frac{8 R^{2}}{d^{2}}\right)^{m}\right)^{n} \\
& =\sum_{n=0}^{\infty}\left(\frac{r}{4} \sum_{m=1}^{M}\left(\frac{8 R^{2}}{d^{2}}\right)^{m}\right)^{n}
\end{aligned}
$$

However the sum over $m$ is dominated by the infinite sum and

$$
\frac{r}{4} \sum_{m=1}^{\infty}\left(\frac{8 R^{2}}{d^{2}}\right)^{m}=\frac{r}{4} \frac{8 R^{2} / d^{2}}{1-8 R^{2} / d^{2}}<\frac{1}{4}
$$

since under our assumption $8 R^{2} / d^{2} \leq 8 r R^{2} / d^{2}<1 / 2$. Then the final sum over $n$ in (141) converges and is bounded in $M$. Hence the sum $\sum_{\left\{n_{m}^{j}\right\}}\left|\mathcal{A}_{r}\right|^{2}$ converges without the restriction $m \leq M$ which is our result.

Remarks. To extend these results to bosonic string theory and define (tree level, genus zero) string theory scattering amplitudes one would have to make a number of modifications which we now list.

1. Replace $X: \mathbb{C}_{\infty} \rightarrow \mathbb{R}$ by $X: \mathbb{C}_{\infty} \rightarrow \mathbb{R}^{d}$ where $d$ is the dimensional of spacetime, preferably $d=26$. The basic covariance is then

$$
<\partial X^{\mu}(z) \partial X^{\nu}\left(z^{\prime}\right)>=-\frac{1}{2} \delta^{\mu \nu}(2 \pi)^{-1}\left(z-z^{\prime}\right)^{-2}
$$

2. Replace the monomials : $\partial^{m_{1}} X^{\mu_{1}}(z) \cdots \partial^{m_{n}} X^{\mu_{n}}(z)$ : by expressions (vertex functions)

$$
: \partial^{m_{1}} X^{\mu_{1}}(z) \cdots \partial^{m_{n}} X^{\mu_{n}}(z) e^{i k \cdot X(\bar{z}, z)}:
$$

The factor $e^{i k \cdot X(\bar{z}, z)}$ with $k \in \mathbb{R}^{d}$ is present to accommodate the center of mass motion.

3. Analytically continue from the Euclidean metric $\delta_{\mu \nu}$ to the Minkowski metric $\eta_{\mu \nu}$. Show that the correlation functions define multilinear functionals on a Hilbert space with a particle interpretation. This will require that the momenta $k$ be on the mass shell and has to deal with the fact that the natural inner products with the Minkowski metric are indefinite.

4. Integrate over the positions of the vertex functions over $\mathbb{C}_{\infty}$. This will involve isolating the singularities that occur.

This program is reasonably well understood for a few low lying states (see Polchinski [6]), but a systematic treatment is lacking 


\section{References}

[1] J. Dimock, Markov quantum fields on a manifold, Rev. Math. Phys 16, 243-255, 2004

[2] J. Dimock, Transition amplitudes and sewing properties for bosons on the Riemann spere, J. Math. Phys. 48, 052308, 1-31, 2007.

[3] G. Felder, J. Frohlich, J. Keller, On the structure of unitary conformal field theory, Commun. Math. Phys. 124, 417-463, 1989

[4] K. Gawedski, Lectures on conformal field theory, in Quantum fields and strings: a course for mathematicians, P. Deligne et. al. eds., American Mathematical Society, Providence, 1999.

[5] D. Pickrell, $P(\phi)_{2}$ quantum field theories and Segal's axioms. (math-ph/0702077)

[6] J. Polchinski, String Theory, Cambridge University Press, Cambridge, 1998.

[7] M. Schottenloher, A mathematical introduction to conformal field theory, Springer, 1997 